\title{
DNA Directed Self-Assembly of Single Walled Carbon Nanotubes into Three-Way Junction Nanostructures
}

\author{
Betul Oruc, ${ }^{\dagger}$ Suleyman Celik, ${ }^{\ddagger}$ Serap Hayat Soytas, ${ }^{\ddagger}$ and Hayriye Unal ${ }^{*} \ddagger$ \\ ${ }^{\dagger}$ Faculty of Engineering and Natural Sciences, Sabanci University, 34956 Istanbul, Turkey \\ ${ }^{\ddagger}$ Sabanci University SUNUM Nanotechnology Research Center, 34956 Istanbul, Turkey
}

Supporting Information

ABSTRACT: Utilization of a self-assembled two-dimensional DNA nanostructure to arrange single-walled carbon nanotubes (SWNTs) into predetermined structures at controllable angles is presented. A specially designed DNA three-way junction (3WJ) composed of three double-stranded DNA arms containing single-stranded overhang sequences was prepared by annealing of partially complementary ssDNA sequences and ultrasonicated with SWNTs, resulting in DNA-3WJ/SWNT hybrid nanostructures. Utilization of DNA-3WJ not only allowed the precise dispersion of SWNTs but also acted as a rigid template for the self-assembly of SWNTs into three-

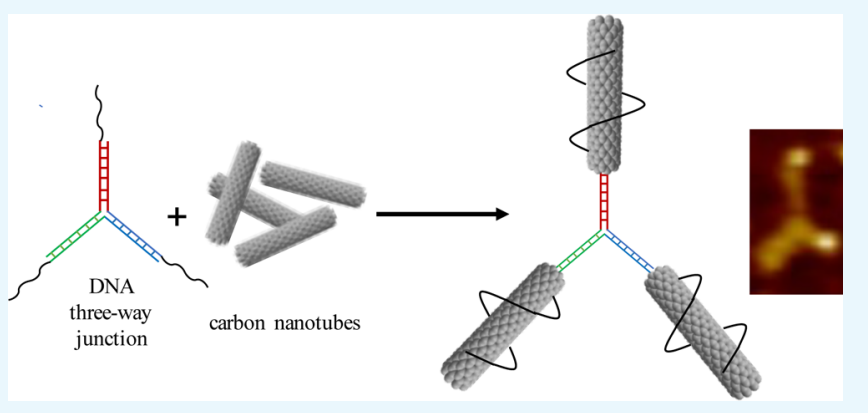
armed junctions at an angle of approximately $120^{\circ}$ to each other as visualized by scanning electron microscopy and atomic force microscopy. Prepared DNA-3WJ/SWNT nanostructures were also demonstrated to have the appropriate binding sites for fluorophores, providing a simple method for the fluorescent labeling of SWNTs. When ssDNA sequences forming the DNA-3WJ are ultrasonicated with SWNTs, followed by annealing of resulting ssDNA wrapped SWNTs, instead of hybrid junctions composed of three SWNT molecules, a web-like structure composed of interconnected SWNT junctions was obtained. The design approaches demonstrated here provide simple methods for the arrangement of SWNTs into desired nanostructures utilizing pre-assembled DNA nanostructures as linkers in aqueous solution through noncovalent interactions which can greatly contribute to efforts along the controlled assembly of SWNTs.

\section{INTRODUCTION}

Single-walled carbon nanotubes (SWNTs) are essential building blocks for the fabrication of nanoscale devices with potential applications in electronics, optics, and biosensing. The main challenge in the utilization of remarkable thermal, electrical, and electronical properties of SWNTs in such nanoscale devices is to precisely control the assembly of SWNTs into desired nanoarchitectures. The ability to direct the positioning of SWNTs at the nanoscale could allow the creation of macroscopic assemblies that parallel the distinct properties of individual nanotubes. Several studies focused on building SWNT junctions as synthesized on surfaces ${ }^{1-5}$ and in solution using postsynthesis methodologies. ${ }^{6-11}$ A promising approach to build controllable SWNT assemblies is the utilization of single-stranded DNA (ssDNA) as information-containing biomolecules. ssDNA is a useful tool for materials chemistry as it can be programmed into complex nanostructures through hybridization and allows both covalent and noncovalent functionalization to build hybrid materials. ${ }^{12,13}$ The fact that ssDNA can form strong $\pi-\pi$ interactions with SWNTs resulting in helical wrapping makes it a useful agent that can be utilized for SWNT dispersion, sorting, and labeling. ${ }^{14}$ Hybrids of ssDNA and SWNTs can be prepared where the ssDNA can act as both the dispersing agent by wrapping the SWNT and also as the linker molecule that joins SWNTs wrapped with ssDNA of complimentary sequences through hybridization. The ability of ssDNA to direct the self-assembly of SWNTs into controlled aggregates or two-dimensional complex geometries in solution or on ssDNA-immobilized surfaces has been demonstrated in a limited number of studies. $^{15-22}$ On the other hand, a facile method to arrange SWNTs into controlled nanostructures utilizing pre-assembled DNA nanostructures that link the self-assembly of SWNTs through in-solution, noncovalent interactions is missing.

Here, we demonstrate the utilization of a specially designed ssDNA three-way junction (3WJ) nanostructure to build branched SWNT building blocks in solution. DNA 3WJ nanostructures possessing linker sequences available for wrapping the SWNT allow the self-assembly of three individual SWCNTs into a Y-junction. Depending on the route of preparation, these SWNT Y-junctions can act as building blocks for the formation of larger networks of SWNTs as well.

\section{RESULTS AND DISCUSSION}

Preparation and Characterization of 3WJ-DNA Nanostructures. The DNA nanostructure utilized to link three

Received: February 20, 2018

Accepted: April 5, 2018

Published: April 12, 2018 

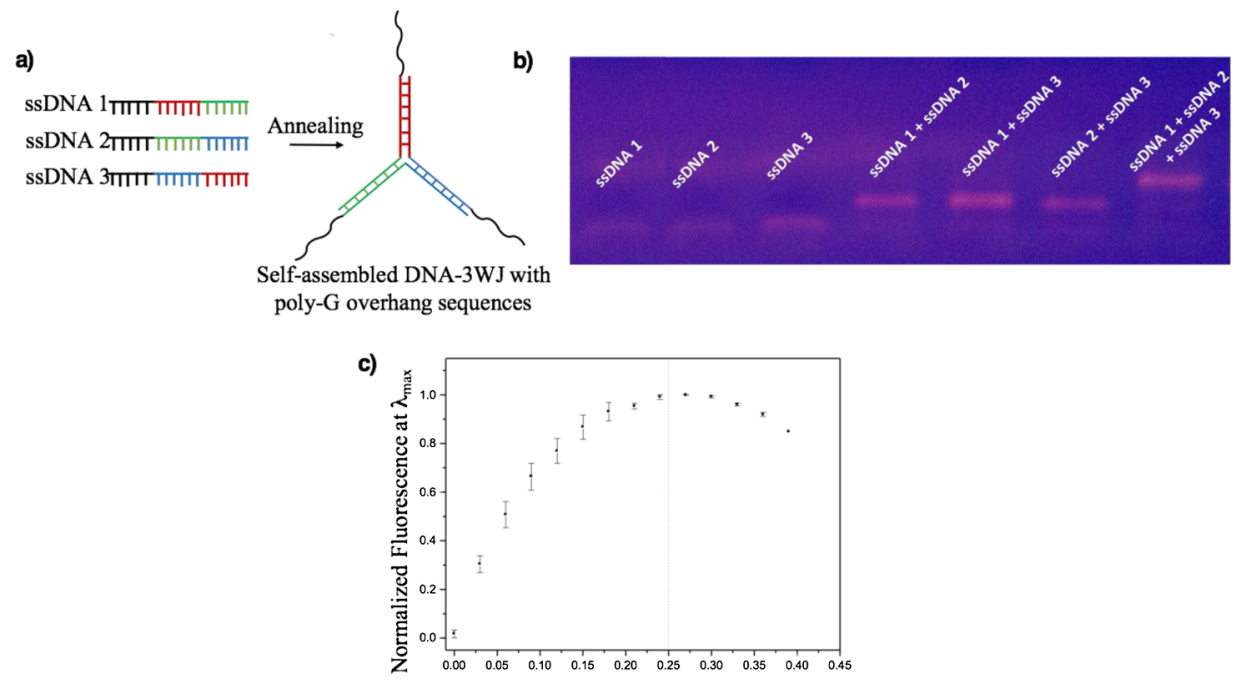

[YOYO-1]/[DNA base]

Figure 1. (a) Schematic of the self-assembly of DNA-3WJ. Same color regions represent complementary sequences, and black regions represent poly-G sequences, (b) visualization of the agarose gel loaded with individual ssDNA strands, binary combinations of ssDNA strands, and a mixture of all three ssDNA strands. All samples were annealed to $95{ }^{\circ} \mathrm{C}$ for $5 \mathrm{~min}$ and cooled in an ice bath, and (c) fluorescence titration curve demonstrating the increase in fluorescence intensity of DNA-3WJ at increasing fluorophore concentrations.

individual SWNT molecules into a Y-junction with a controlled angle through noncovalent binding interactions is a DNA-3WJ. The $3 \mathrm{WJ}$ is formed by the self-assembly of three ssDNA strands with partially complementary sequences through annealing, resulting in a nanostructure with three arms consisting of double-stranded DNA and noncomplementary overhang sequences for wrapping the SWNT (Figure 1a). The overhang is designed to be a poly-G sequence consisting of 13 guanines which was previously demonstrated to show a strong affinity toward wrapping SWNTs. ${ }^{23}$ The successful assembly of the $3 \mathrm{WJ}$ was demonstrated with agarose gel electrophoresis (Figure 1b). When all three ssDNA strands were annealed, the corresponding band migrates slower than bands corresponding to individual strands and to the products obtained from annealing two of the ssDNA strands using the same conditions. This result demonstrated the successful formation of the DNA3WJ nanostructure which migrates slower than control products because of its larger molecular weight.

The formation of the $3 \mathrm{WJ}$ nanostructure exactly as designed was further confirmed by measuring the number of base pairs formed by conducting fluorophore titration experiments. Aliquots of an asymmetrical cyanine dimer, YOYO-1, that is known to bind to DNA base pairs at a ratio of 1:4 was titrated into a DNA-3WJ solution of a known concentration, and the increase in fluorescence was monitored at increasing fluorophore concentrations. Figure 1c shows that the fluorescence intensity increased with the increasing fluorophore concentration as YOYO-1 molecules intercalate between the base pairs of $3 \mathrm{WJ}$ and light up. At a certain point, the fluorescence intensity levels off, indicating that all binding sites on the nanostructure were saturated with the fluorophore. The fact that this saturation occurred when the ratio of the YOYO-1 concentration to the theoretical number of base pairs is 0.25 , as expected, confirmed the formation of the $3 \mathrm{WJ}$ nanostructure as designed.

Preparation and Characterization of Hybrids of DNA3WJ and SWNTs. To assemble SWNTs into 3WJ nanostructures, they were ultrasonicated in an aqueous solution of the prepared DNA-3WJ, followed by ultracentrifugation to remove any residues that are not dispersed (Figure 2a). While SWNTs of the same concentration were not dispersed in water using

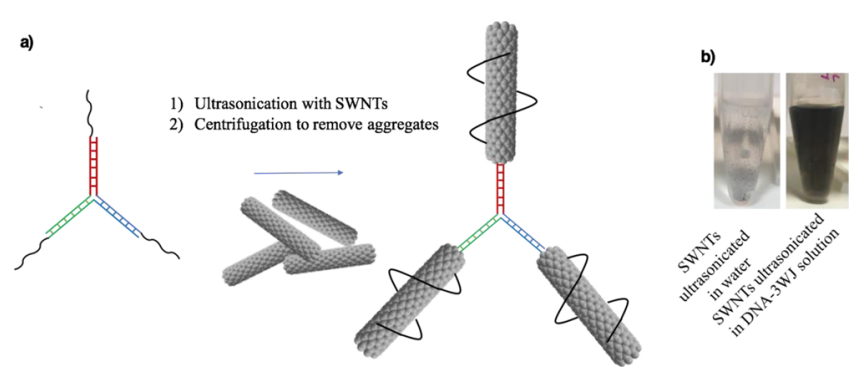

Figure 2. (a) Schematic representation of the formation of DNA3WJ/SWNT hybrid nanostructures and (b) photographs of SWNTs ultrasonicated in water (left) and SWNTs ultrasonicated in DNA-3WJ solution.

the same ultrasonication conditions, SWNTs ultrasonicated in DNA-3WJ solution were finely dispersed, indicating that SWNTs were noncovalently interacting with the DNA-3WJ, leading to the formation of a hybrid nanostructure (Figure $2 b$ ). As a control experiment to confirm that DNA-3WJ nanostructures survived the high-energy ultrasonication process, agarose gel migration of samples before and after the ultrasonication was compared and shown to have the same mobility, demonstrating that the DNA-3WJ template stays intact during the ultrasonication of SWNTs with DNA (data not shown).

The formation of DNA-3WJ/SWNT hybrid structures was further characterized with atomic force microscopy (AFM) and scanning electron microscopy (SEM) to confirm that SWNTs are assembled into junctions with a controlled angle. Figure 3a shows representative AFM images of DNA-3WJ/SWNT hybrids. As seen on the images, the DNA-3WJ template guided the assembly of three individual SWNT molecules into one hybrid nanostructure, leading to three-armed SWNTs where the angle at their junction point is approximately $120^{\circ}$. The fact that the DNA-3WJ nanostructure is composed of three partially 
a)

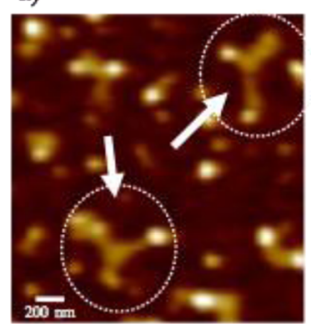

b)

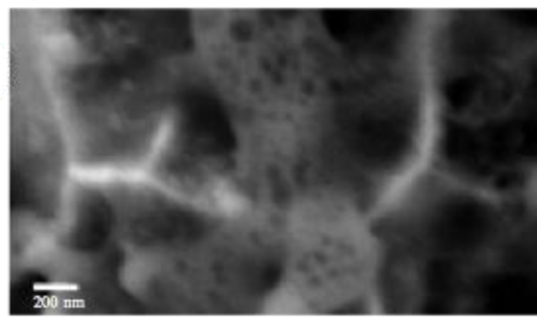

Figure 3. (a) AFM topographical image of DNA-3WJ/SWNT hybrid nanostructures and (b) cross-sectional analysis of representative topographical AFM (a) and SEM (b) images of DNA-3WJ/SWNT hybrid nanostructures.

complementary strands that form double-stranded DNA arms makes it a rigid template that controls the angle at which the carbon nanotubes join and form the hybrid three-armed SWNT nanostructure. Cross-sectional AFM height analysis confirms that these Y-junction nanostructures are actually composed of SWNTs as the height of these nanostructures coincides with the height of individual SWNTs imaged under the same conditions (Supporting Information Figure S1). While Yjunctions of SWNTs were visible on the representative AFM image, individual SWNTs were present as well, indicating that not all SWNTs were able to self-assemble into DNA-3WJ/ SWNT nanostructures. We believe that manipulation of DNA3WJ and SWNT concentrations along with sonication and annealing conditions can improve the yield of DNA-3WJ/ SWNT formation. Similar to AFM images, DNA-3WJtemplated branched SWNT nanostructures were also visible on SEM images (Figure 3b). Microscopy images obtained confirmed that the design and the method applied in this work allowed the DNA-3WJ-templated assembly of SWNTs into three-armed junctions.

DNA-3WJ nanostructures not only acted as templates that facilitate the self-assembly of SWNTs into junctions, they were also shown to provide a relatively better dispersion quality compared to linear ssDNA strands that form the DNA-3WJ. We utilized agarose gel electrophoresis to demonstrate the relative dispersion qualities of SWNT samples, as improved quality of dispersion of SWNTs will decrease the particle size and make it easier for SWNTs to enter the gel and migrate. Figure 4a shows a comparison of migration of ssDNA3/SWNT and $3 \mathrm{WJ} / \mathrm{SWNT}$ hybrids in an agarose gel. While both hybrids entered the gel, $3 \mathrm{WJ} / \mathrm{SWNT}$ hybrids migrated significantly a)

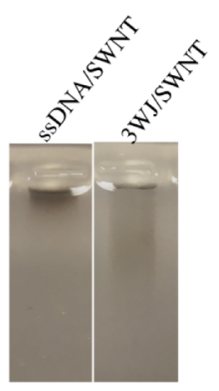

b)

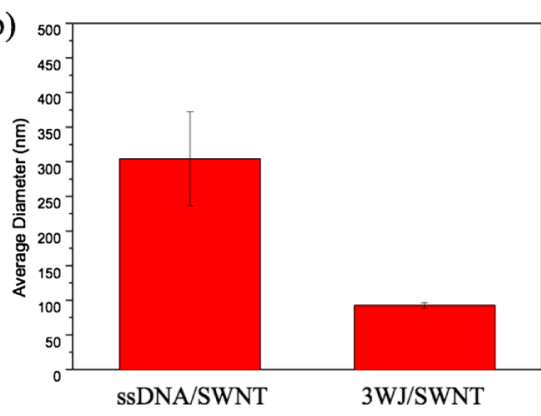

Figure 4. (a) Visualization of an agarose gel loaded with equal amounts of ssDNA/SWNT (left) and DNA-3WJ/SWNT (right) and (b) average hydrodynamic diameters of ssDNA/SWNT and DNA3WJ/SWNT hybrids measured by DLS. faster than ssDNA/SWNT hybrids on the agarose gel, indicating that SWNTs were dispersed into smaller particle sizes in the DNA-3WJ solution than the linear ssDNA. Dynamic light scattering (DLS) characterization of both samples reporting the average hydrodynamic diameter of SWNTs further confirmed that $3 \mathrm{WJ}$ nanostructures provided better dispersion quality compared to a linear ssDNA (Figure $4 b$ ). The particle size of SWNTs dispersed using the DNA 3WJ were measured to be significantly smaller than the particle size of the same amount of SWNTs dispersed using a linear equivalent, one of the ssDNA strands forming the DNA-3WJ. Apparently, the dendrimeric form of the DNA-3WJ binding sites which allows one DNA-3WJ molecule to wrap three individual SWNT molecules increases the dispersion efficiency.

We further characterized DNA-3WJ/SWNT nanostructures for their potential for being fluorescently labeled. Fluorescent labeling of nanoparticles provides a great variety of opportunities for the utilization of nanoparticles in biological and sensing applications. Fluorescent labeling of SWNTs with fluorophores has been of particular interest because the inherent fluorescence emission of SWNTs is of very low efficiency and is limited to the near-infrared region of the spectrum. The main obstacle that limits the fluorescent labeling of SWNTs with fluorophores is the fact that fluorophores in close proximity to the SWNT surface are mostly quenched, as SWNTs act as electron and energy acceptors. ${ }^{24,25}$ The design of DNA-3WJ/SWNT nanohybrids reported here allows the labeling of the core DNA-3WJ with fluorophores which results in indirect labeling of SWNTs. To investigate whether DNA$3 \mathrm{WJ} / \mathrm{SWNTs}$ can be labeled with fluorophores, we chose an asymmetric cyanine dye, YOYO-1 which lights up when it intercalates between the base pairs of double-stranded DNA. Figure 5 shows the fluorescence spectrum of a solution of DNA-3WJ/SWNTs, to which a saturating amount of YOYO-1 was added.

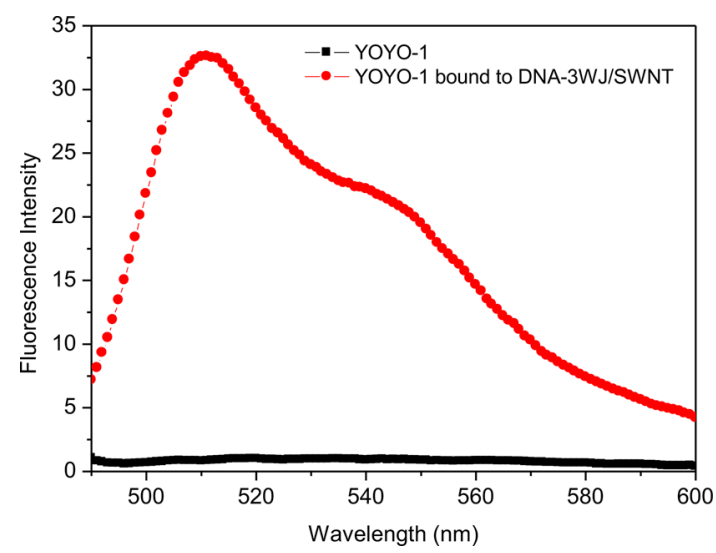

Figure 5. Fluorescence spectra of equal amounts of YOYO-1 (i) in water (black squares) and (ii) bound to DNA-3WJ/SWNT (red circles).

While YOYO-1 was not fluorescent when it is free in aqueous solution, DNA-3WJ/SWNT nanohybrids, to which the same amount of YOYO-1 was added, had a strong fluorescence intensity. Utilization of a DNA nanostructure not only allowed the self-assembly of SWNTs into a controlled branched nanostructure, it also acted as a functional group that enables the fluorescent labeling of SWNTs. 
An alternative design approach to prepare DNA-3WJ/SWNT nanohybrids was also investigated where individual ssDNA strands designed to form the $3 \mathrm{WJ}$ were ultrasonicated with SWNTs first, followed by the annealing of ssDNA-wrapped SWNTs (Figure 6). While we hypothesized that a similar DNA-

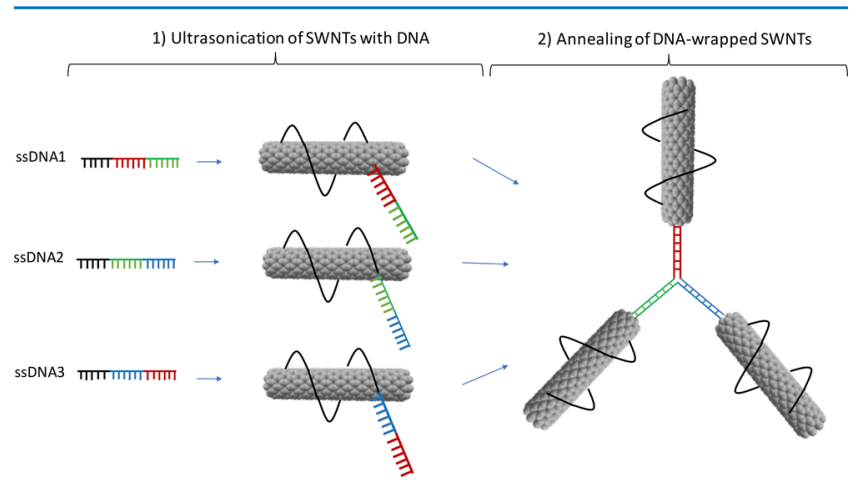

Figure 6. Schematic representation of the hypothesized mechanism of formation of DNA-3WJ/SWNT nanostructures with the postannealing method.

3WJ/SWNT nanohybrid structure may form using this postannealing preparation method, characterization of the prepared product predicted a different nanostructure.

Prepared DNA-3WJ/SWNTs were characterized with agarose gel electrophoresis. While nanostructures formed by the preannealing method entered the gel and migrated, nanostructures formed by the postannealing method were not able to enter the gel (Figure 7a). DNA-3WJ/SWNT nanostructures prepared with the postannealing method remained in the loading well, indicating a significantly larger molecular weight that limits the mobility of the nanostructure within the gel. Similarly, characterization of the average hydrodynamic diameter of the nanostructures formed by the postannealing method predicted a bulky structure with a diameter that is several folds larger than the DNA-3WJ/SWNT nanohybrids formed by the preannealing method (Figure $7 \mathrm{~b}$ ). These results indicated that the postannealing method resulted in a network of DNA-3WJ-linked SWNTs instead of individual three-armed SWNT hybrids. Microscopic characterization of resulting nanostructures further confirmed the formation of such a network of SWNTs (Figure 8a,b, Supporting
Information Figure S2). SWNTs, mostly self-assembled into junctions, appeared to be interconnected to each other forming a web-like structure composed of hexagons. When the twodimensional planar $3 \mathrm{WJ}-\mathrm{SWNT}$ nanostructures were linked at different angles, a three-dimensional network of SWNTs was possibly formed, as also visible on the microscopy images. A possible mechanism of formation of this SWNT network structure is demonstrated in Figure 8c. If multiple ssDNA strands wrap the same SWNT through the poly-G regions during the ultrasonication process, then SWNTs with multiple overhang sequences can form, which will be linked into a network when the complementary overhang sequences hybridize during the annealing step. When the preannealing method is applied, on the other hand, the rigid and bulky nature of the 3WJ implies a steric hindrance that prevents the wrapping of SWNTs by multiple $3 \mathrm{WJ}$ nanostructures which is not the case when SWNTs are ultrasonicated with linear ssDNA strands. Thus, we have demonstrated that the order of addition of the ssDNA strands that form the DNA-3WJ nanostructure and SWNTs can determine the structure of the DNA-3WJ/SWNT nanostructures. SWNTs self-assembled into Y-junctions or into a network composed of Y-junctions can be obtained in a controllable fashion by applying different methods.

\section{CONCLUSIONS}

We demonstrated a simple method to prepare self-assembled SWNT nanostructures where three individual SWNT molecules are linked into a junction at a controllable angle by the utilization of a two-dimensional self-assembled DNA-3WJ nanostructure. While the DNA-3WJ was demonstrated to be a relatively better dispersing agent than a linear ssDNA analog, it also acted as an effective template for the arrangement of SWNTs into a desired nanostructure. The design of the DNA3WJ/SWNT hybrids also allows the effective labeling of SWNTs with fluorophores. With a slight modification of the preparation method where the order of addition of components is changed, these SWNT junctions can be interconnected, resulting in a web-like network of SWNTs. Methods reported here provide an effective approach for the self-assembly of SWNTs in solution, utilizing self-assembled DNA nanostructures and can be applied to various other DNA nanostructures resulting in SWNT assemblies of different structures and dimensions.

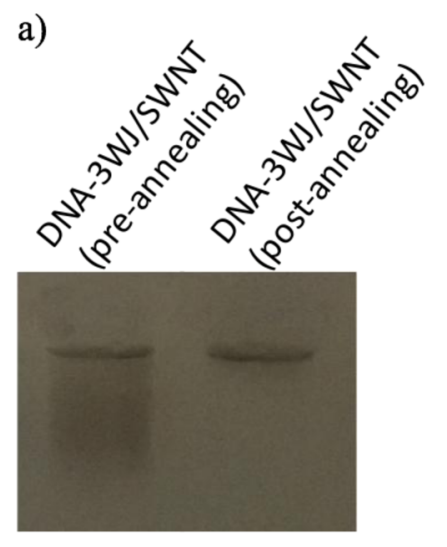

b)

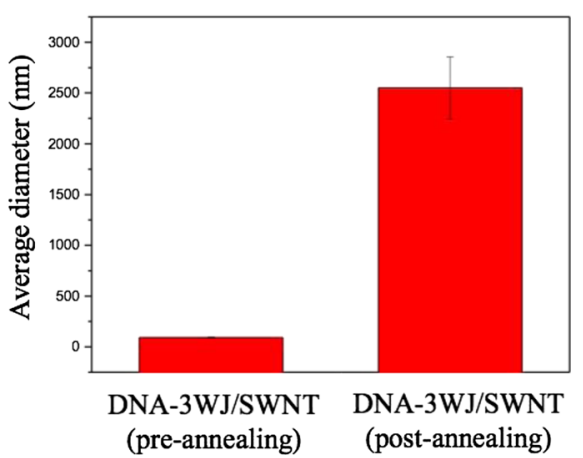

Figure 7. (a) Visualization of an agarose gel loaded with DNA-3WJ/SWNT hybrid nanostructures prepared with preannealing (left) and postannealing (right) methods. (b) Average hydrodynamic diameters of DNA-3WJ/SWNT nanostructures prepared with preannealing (left) and postannealing (right) methods. 


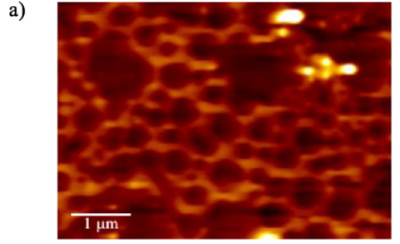

b)

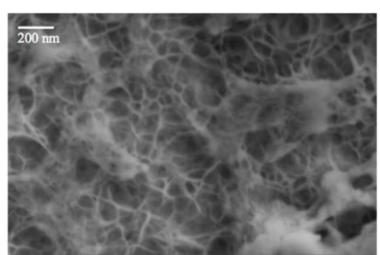

c)

1) Ultrasonication of SWNTs 2) Annealing of DNA-wrapped with ssDNA SWNTs

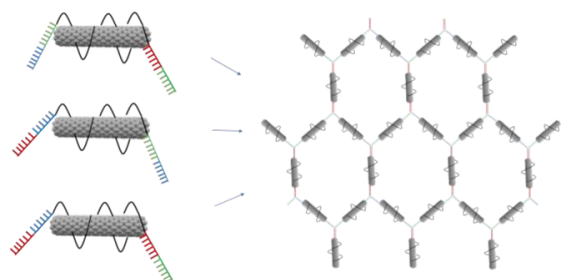

Figure 8. (a) Representative AFM image of DNA-3WJ/SWNT nanostructures prepared with the postannealing method. (b) Representative SEM image of DNA-3WJ/SWNT nanostructures prepared with the postannealing method. (c) Schematic representation of the potential mechanism of formation of the network structure when the postannealing method is used.

\section{EXPERIMENTAL SECTION}

Materials. The powder form of SWNTs with 1-2 nm diameter, $500 \mathrm{~nm}$ length, and $60 \%$ purity was purchased from Nanostructured and Amorphous Materials Inc. in Los Alamos, New Mexico, USA. ssDNA strands to prepare DNA-3WJ nanostructures were synthesized by Sentromer DNA Technologies, Istanbul, Turkey. The sequences of ssDNA used in this study are listed in Table 1. Asymmetrical cyanine dye,

Table 1. Sequences of ssDNA Utilized for the Self-Assembly of DNA-3WJ

\begin{tabular}{ccc} 
ssDNA & \multicolumn{1}{c}{ sequence } & $\begin{array}{c}\text { base } \\
\text { number }\end{array}$ \\
ssDNA1 & $\begin{array}{c}\text { GGG GGG GGG GGG GCA GGT GGC GAG AGC } \\
\text { GAC GAT CCA T }\end{array}$ & 37 \\
ssDNA2 & $\begin{array}{c}\text { GGG GGG GGG GGG GAT GGA TCG TCG CAG } \\
\text { AGT TGA CCG G }\end{array}$ & 37 \\
ssDNA3 & $\begin{array}{c}\text { GGG GGG GGG GGG GCC GGT CAA CTC TTC } \\
\text { TCG CCA CCT G }\end{array}$ & 37 \\
\hline
\end{tabular}

YOYO-1 (Y3601), was purchased from Life Technologies. The gel was stained with GelRed by Biotium, USA. DNA-3WJ/ SWCNT nanohybrids were filtered with Amicon Ultra $0.5 \mathrm{~mL}$ centrifugal filters with $30 \mathrm{k}$ membrane cutoff by EMD Millipore, Darmstadt, Germany.

Preparation and Characterization of DNA-3WJ. To prepare the DNA-3WJ nanostructure, an aqueous solution containing $1 \mu \mathrm{M}$ of each ssDNA1, ssDNA2, and ssDNA3 in a buffer ( $100 \mathrm{mM} \mathrm{NaCl}, 10 \mathrm{mM}$ Tris, and $0.1 \mathrm{mM}$ EDTA) was prepared and annealed by heating to $95{ }^{\circ} \mathrm{C}$ for $5 \mathrm{~min}$ and cooling in an ice bath.

Visualization of the formation of DNA-3WJ was performed by agarose gel electrophoresis. Samples $(12.5 \mu \mathrm{L})$ mixed with $2.5 \mu \mathrm{L} 6 \times$ glycerol loading dye were loaded into a $1 \%$ agarose gel prestained with GelRed nucleic acid stain. The gel was run in $1 \times$ TBE buffer ( $89 \mathrm{mM}$ tris(hydroxymethyl)aminomethane, $89 \mathrm{mM}$ boric acid, and $2 \mathrm{mM}$ EDTA in distilled water). The Bio-Rad Gel Doc EZ system was used to visualize the gel.

The titration experiment to confirm the formation of DNA$3 \mathrm{WJ}$ was conducted on a Cary Eclipse Fluorescence Spectrophotometer. Aliquots of $50 \mu \mathrm{M}$ YOYO-1 solution were titrated into a solution containing $0.25 \mu \mathrm{M}$ DNA-3WJ. The intensity at fluorescence maximum $(510 \mathrm{~nm})$ was monitored at increasing YOYO-1 concentrations, and the saturation of DNA-3WJ with fluorophores was reached when the intensity levels off.

Preparation of DNA-3WJ/SWNT Nanohybrids. To prepare $3 \mathrm{WJ}$-DNA/SWNT nanohybrids with the preannealing method, a dispersal solution composed of $0.1 \mathrm{mg} \mathrm{mL}^{-1}$ SWNT and $1 \mu \mathrm{M}$ annealed DNA-3WJ was prepared and sonicated with a microprobe (QSonica, Q700) for $30 \mathrm{~min}$ at a power of $5 \mathrm{~W}$ with $4 \mathrm{~s}$ pulse on and $4 \mathrm{~s}$ pulse off time in ice. The dispersion was centrifuged at $14000 \mathrm{rpm}$ for $5 \mathrm{~min}$. The black colored supernatant was removed into a clean Eppendorf tube.

To prepare $3 \mathrm{WJ}-\mathrm{SWNT}$ nanohybrids with the postannealing method, each ssDNA was sonicated with SWNTs separately. A solution $(0.5 \mathrm{~mL})$ containing $0.1 \mathrm{mg} \mathrm{mL}^{-1} \mathrm{CNTs}$ and $1 \mu \mathrm{M}$ of each ssDNA in water was sonicated for $30 \mathrm{~min}$ with $4 \mathrm{~s}$ pulse on and $4 \mathrm{~s}$ pulse off time in ice. Following the sonication, equal volumes of all dispersion solutions were mixed and annealed by heating to $95{ }^{\circ} \mathrm{C}$ for $5 \mathrm{~min}$ and cooling in an ice bath. The dispersion was centrifuged to remove the unbound SWNTs at $14000 \mathrm{rpm}$ for $5 \mathrm{~min}$, and the supernatant was taken into a clean Eppendorf tube. Any unbound ssDNA was removed using Amicon Ultra filter devices by following the instructions of the filter device.

Characterization of 3WJ-DNA/SWNT Nanohybrids. Atomic Force Microscopy. The supramolecular assemblies of DNA-3WJ/SWNT nanohybrids were characterized by AFM in a dynamic mode (ezAFM, Nanomagnetics Instruments). To perform the experiment on an atomically flat surface, the mica sheet was cleaved with the scotch tape a few times. Samples were diluted 1:10 with deionized water. Nanohybrids $(20 \mu \mathrm{L})$ were deposited onto the freshly cleaved mica sheet for $15 \mathrm{~min}$ at room temperature. Following the deposition, the surface was rinsed with deionized water and air-dried.

Scanning Electron Microscopy. DNA-3WJ/SWNT nanohybrids were analyzed with a Zeiss Leo Supra 35 scanning electron microscope using a secondary electron detector. Nanohybrids were diluted 1:10 and 1:200 with distilled water for preannealed and postannealed samples, respectively. Nanohybrids $(10 \mu \mathrm{L})$ were dropped onto an aluminum SEM stub and then air-dried for $2 \mathrm{~h}$. DNA-3WJ/SWCNT nanohybrids prepared with the preannealing method were visualized at an accelerating voltage of $4 \mathrm{kV}$ and a working distance of 6 $\mathrm{mm}$. DNA-3WJ/SWCNT nanohybrids prepared with the postannealing method were visualized at an accelerating voltage of $3 \mathrm{kV}$ and a working distance of $7 \mathrm{~mm}$. 
Agarose Gel Electrophoresis. Visualization of the mobility of DNA-3WJ/SWNT nanohybrids was performed by agarose gel electrophoresis. Samples $(12.5 \mu \mathrm{L})$ mixed with $2.5 \mu \mathrm{L}$ of $1: 3$ glycerol: water solution were loaded into a $1 \%$ agarose gel, and the gel was run in $1 \times \mathrm{TBE}$. The gel was photographed without any staining.

Fluorescence Spectroscopy. The fluorescence spectrum of 3WJ-DNA/SWNT saturated with YOYO-1 was obtained by performing a titration experiment. Aliquots of a $10 \mu \mathrm{M}$ YOYO1 solution were titrated into a solution containing $0.25 \mu \mathrm{M}$ $3 \mathrm{WJ}-\mathrm{DNA} / \mathrm{SWNT}$. The intensity at fluorescence maximum $(510 \mathrm{~nm})$ was monitored at increasing YOYO-1 concentrations, and the saturation of the nanohybrid with fluorophores was reached when the intensity levels off.

Dynamic Light Scattering. Samples were analyzed in a quartz cuvette at $25^{\circ} \mathrm{C}$. Nanohybrids were diluted 1:200 with distilled water for measurements. The particle size analysis was performed for 10 cycles with 3 measurements that were used to determine the mean particle size.

\section{ASSOCIATED CONTENT}

\section{S Supporting Information}

The Supporting Information is available free of charge on the ACS Publications website at DOI: 10.1021/acsomega.8b00306.

Cross-sectional AFM height analysis of DNA/SWNT nanostructures and 3-D AFM image of DNA-3WJ/ SWNT nanostructures prepared with the postannealing method (PDF)

\section{AUTHOR INFORMATION}

\section{Corresponding Author}

*E-mail: hunal@sabanciuniv.edu (H.U.).

\section{ORCID 1}

Hayriye Unal: 0000-0002-9090-2440

Notes

The authors declare no competing financial interest.

\section{ACKNOWLEDGMENTS}

The funding for this work was provided by the Sabanci University Personal Research Fund.

\section{REFERENCES}

(1) $\mathrm{Xu}$, J.; Li, J.; Papadopoulos, C. Nanoelectronics: Growing YJunction Carbon Nanotubes. Nature 1999, 402, 253-254.

(2) Osváth, Z.; Koós, A. A.; Horváth, Z. E.; Gyulai, J.; Benito, A. M.; Martínez, M. T.; Maser, W. K.; Biró, L. P. Arc-Grown Y-Branched Carbon Nanotubes Observed by Scanning Tunneling Microscopy (STM). Chem. Phys. Lett. 2002, 365, 338-342.

(3) Ouyang, M.; Huang, J.-L.; Cheung, C. L.; Lieber, C. M. Atomically Resolved Single-Walled Carbon Nanotube Intramolecular Junctions. Science 2001, 291, 97-100.

(4) Jin, C.; Suenaga, K.; Iijima, S. Plumbing Carbon Nanotubes. Nat. Nanotechnol. 2007, 3, 17-21.

(5) Luo, C.; Liu, L.; Jiang, K.; Zhang, L.; Li, Q.; Fan, S. Growth Mechanism of Y-Junctions and Related Carbon Nanotube Junctions Synthesized by Au-Catalyzed Chemical Vapor Deposition. Carbon 2008, 46, 440-444.

(6) Sano, M.; Kamino, A.; Okamura, J.; Shinkai, S. Ring Closure of Carbon Nanotubes. Science 2001, 293, 1299.

(7) Chiu, P. W.; Duesberg, G. S.; Dettlaff-Weglikowska, U.; Roth, S. Interconnection of Carbon Nanotubes by Chemical Functionalization. Appl. Phys. Lett. 2002, 80, 3811-3813.
(8) Weizmann, Y.; Chenoweth, D. M.; Swager, T. M. Addressable Terminally Linked DNA-CNT Nanowires. J. Am. Chem. Soc. 2010, 132, 14009-14011.

(9) Palma, M.; Wang, W.; Penzo, E.; Brathwaite, J.; Zheng, M.; Hone, J.; Nuckolls, C.; Wind, S. J. Controlled Formation of Carbon Nanotube Junctions via Linker-Induced Assembly in Aqueous Solution. J. Am. Chem. Soc. 2013, 135, 8440-8443.

(10) Zhu, J.; McMorrow, J.; Crespo-Otero, R.; Ao, G.; Zheng, M.; Gillin, W. P.; Palma, M. Solution-Processable Carbon Nanoelectrodes for Single-Molecule Investigations. J. Am. Chem. Soc. 2016, 138, 29052908.

(11) Roberts, G. S.; Singjai, P. Joining Carbon Nanotubes. Nanoscale 2011, 3, 4503.

(12) Zhang, F.; Nangreave, J.; Liu, Y.; Yan, H. Structural DNA Nanotechnology: State of the Art and Future Perspective. J. Am. Chem. Soc. 2014, 136, 11198-11211.

(13) Yang, D.; Hartman, M. R.; Derrien, T. L.; Hamada, S.; An, D.; Yancey, K. G.; Cheng, R.; Ma, M.; Luo, D. DNA Materials: Bridging Nanotechnology and Biotechnology. Acc. Chem. Res. 2014, 47, 19021911.

(14) Sanchez-Pomales, G.; Pagan-Miranda, C.; Santiago-Rodriguez, L. R. C. DNA-Wrapped Carbon Nanotubes: From Synthesis to Applications. In Carbon Nanotubes; Mauricio Marulanda, J., Ed.; InTech, 2010.

(15) Maune, H. T.; Han, S.-p.; Barish, R. D.; Bockrath, M.; Goddard, W. A., III; Rothemund, P. W. K.; Winfree, E. Self-Assembly of Carbon Nanotubes into Two-Dimensional Geometries Using DNA Origami Templates. Nat. Nanotechnol. 2010, 5, 61-66.

(16) Cheng, E.; Li, Y.; Yang, Z.; Deng, Z.; Liu, D. DNA-SWNT Hybrid Hydrogel. Chem. Commun. 2011, 47, 5545-5547.

(17) Chen, Y.; Liu, H.; Ye, T.; Kim, J.; Mao, C. DNA-Directed Assembly of Single-Wall Carbon Nanotubes. J. Am. Chem. Soc. 2007, 129, 8696-8697.

(18) Xu, P. F.; Noh, H.; Lee, J. H.; Cha, J. N. DNA Mediated Assembly of Single Walled Carbon Nanotubes: Role of DNA Linkers and Annealing. Phys. Chem. Chem. Phys. 2011, 13, 10004.

(19) Han, S.-p.; Maune, H. T.; Barish, R. D.; Bockrath, M.; Goddard, W. A. DNA-Linker-Induced Surface Assembly of Ultra Dense Parallel Single Walled Carbon Nanotube Arrays. Nano Lett. 2012, 12, 11291135.

(20) McMorrow, J.; Freeley, M.; Palma, M. DNA-Wrapped SingleWalled Carbon Nanotube Assemblies. Ind. Eng. Chem. Res. 2017, 56, 5302-5308.

(21) Li, S.; He, P.; Dong, J.; Guo, Z.; Dai, L. DNA-Directed SelfAssembling of Carbon Nanotubes. J. Am. Chem. Soc. 2005, 127, 14.

(22) Lu, Y.; Yang, X.; Ma, Y.; Du, F.; Liu, Z.; Chen, Y. SelfAssembled Branched Nanostructures of Single-Walled Carbon Nanotubes with DNA as Linkers. Chem. Phys. Lett. 2005, 419, 390.

(23) Albertorio, F.; Hughes, M. E.; Golovchenko, J. A.; Branton, D. Base Dependent DNA-carbon Nanotube Interactions: Activation Enthalpies and Assembly-disassembly Control. Nanotechnology 2009, 20, 395101.

(24) Zhu, Z.; Yang, R.; You, M.; Zhang, X.; Wu, Y.; Tan, W. SingleWalled Carbon Nanotube as an Effective Quencher. Anal. Bioanal. Chem. 2010, 396, 73-83.

(25) Chiu, C. F.; Dementev, N.; Borguet, E. Fluorescence Quenching of Dyes Covalently Attached to Single-Walled Carbon Nanotubes. J. Phys. Chem. A 2011, 115, 9579-9584. 